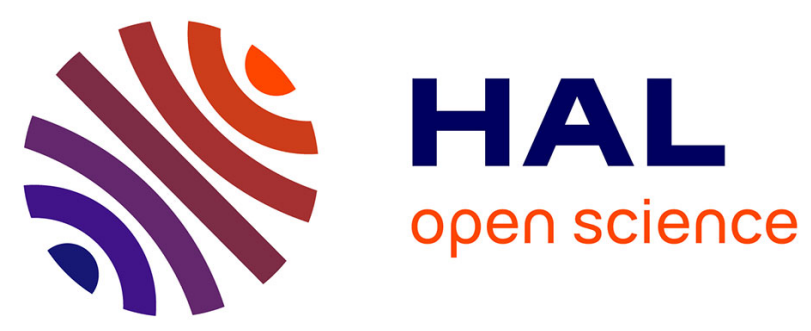

\title{
Electrophysiological Activity Evoked by Direct Electrical Stimulation of the Human Brain: Interest of the P0 Component
}

\author{
Anthony Boyer, Hugues Duffau, Marion Vincent, Sofiane Ramdani, \\ Emmanuel Mandonnet, David Guiraud, François Bonnetblanc
}

\section{To cite this version:}

Anthony Boyer, Hugues Duffau, Marion Vincent, Sofiane Ramdani, Emmanuel Mandonnet, et al.. Electrophysiological Activity Evoked by Direct Electrical Stimulation of the Human Brain: Interest of the P0 Component. 2018 40th Annual International Conference of the IEEE Engineering in Medicine and Biology Society, Jul 2018, Honolulu, HI, United States. pp.2210-2213, 10.1109/EMBC.2018.8512733 . hal-01873027

\section{HAL Id: hal-01873027 \\ https://hal.science/hal-01873027}

Submitted on 12 Sep 2018

HAL is a multi-disciplinary open access archive for the deposit and dissemination of scientific research documents, whether they are published or not. The documents may come from teaching and research institutions in France or abroad, or from public or private research centers.
L'archive ouverte pluridisciplinaire HAL, est destinée au dépôt et à la diffusion de documents scientifiques de niveau recherche, publiés ou non, émanant des établissements d'enseignement et de recherche français ou étrangers, des laboratoires publics ou privés. 


\title{
Electrophysiological Activity Evoked by Direct Electrical Stimulation of the Human Brain: Interest of the P0 Component*
}

\author{
Anthony Boyer, Hugues Duffau, Marion Vincent, Sofiane Ramdani, Emmanuel Mandonnet, David \\ Guiraud, Member, IEEE EMBS and Francois Bonnetblanc
}

\begin{abstract}
Direct electrical stimulation (DES) at $60 \mathrm{~Hz}$ is used clinically to perform real-time functional mapping of the brain and guide tumor resection during wide-awake neurosurgery. The electrophysiological effects of DES remain by far unknown, both locally and remotely. In this study, by lowering the DES frequency to $9 \mathrm{~Hz}$ and by using differential recording of electro-corticographic signals to improve the focality, we were able to observe that the amplitude of the initial P0 component of the direct cortical response increased when the inter-electrode distance was increased and the pulse width was decreased. This result strongly suggests that larger neural elements, including somas and axons of pyramidal neurons buried in deeper layers of the cortical column, are activated. Their activation produce the observed P0 component, which results from the synchronized summation of action potentials triggered by DES. Interestingly, the early P0 component was not observed during the usual $60 \mathrm{~Hz}$ DES. The study of the PO component and subsequent evoked potentials may help decipher the effects of DES on the stimulated cortical column and identify the activation of underlying white matter fibers. This is crucial to better understand the electrophysiological diffusion of DES, especially at higher frequencies (e.g. $60 \mathrm{~Hz}$ ).
\end{abstract}

\section{INTRODUCTION}

Direct Electrical Stimulation (DES) is used to perform real-time functional mapping of the brain while the patient is awake. The intraoperative use of DES has been widely recommended for the neurosurgery of slow-growing and infiltrative brain tumors, to guide the resection [1]. Generally, DES is applied at $60 \mathrm{~Hz}$ (biphasic stimuli, single pulse duration of $1 \mathrm{~ms}$, intensities from 2 to $6 \mathrm{~mA}$, train duration of 1 to $5 \mathrm{~s}$, and under local anesthesia) and generates transient behavioral/cognitive disturbances, allowing the real-time identification of both cortical areas and subcortical white matter pathways which are essential to the considered

*Research supported by the LabEx NUMEV project ( $\mathrm{n}^{\circ}$ ANR-10LABX-20) funded by the French government's "Investissements d'Avenir" program managed by the French National Research Agency (ANR) and complementary grants from the Institut Universitaire de France and INSERM laboratory (U1093).

A. Boyer, M. Vincent, D. Guiraud and F. Bonnetblanc are with the National Institute for Research in Informatics and Automatics (INRIA), 34095 Montpellier, France (corresponding authors; e-mail: francois.bonnetblanc@inria.fr and david.guiraud@inria.fr).

S. Ramdani is with CNRS-University of Montpellier, LIRMM Interactive Digital Human group, UMR5506, Montpellier, France (e-mail: sofiane.ramdani@umontpellier.fr).

H. Duffau is with the Department of Neurosurgery, University of Montpellier, and the Institute of Neurosciences, INSERM U-1051, Hopital Saint-Eloi, 34095 Montpellier, France (e-mail: h-duffau@ chumontpellier.fr).

E. Mandonnet is with the Department of Neurosurgery, Hopital Lariboisière, Paris, France (e-mail: emmanuel.mandonnet@ aphp.fr). function execution. Its use lowers the probability of resecting essential functional areas, near or within the tumor. However, the electrophysiological effects of DES remain poorly understood, both locally and remotely, and thus require further investigations [2, 3]. In this perspective, the recording of Evoked Potentials (EPs) could be used to study the conductive and integrative properties of the neural ensembles being directly stimulated and/or indirectly affected by DES. Three different types of potentials evoked by DES should be distinguished: Cortical evoked-potential (called Direct Cortical Response, DCR), when recording the cortex activity at the stimulation site. Cortico-Cortical Evoked-Potential (CCEP), i.e. recording the cortex activity at a distant site from the stimulation site. CCEPs are elicited by a physiological propagation through white matter tracts from the locally stimulated area towards the distal area. AxonoCortical Evoked-Potentials (ACEP), when a cortical response is recorded from a stimulation site located within the white matter.

The DCR is an EP recorded in the immediate vicinity (distance to the stimulation site $<10 \mathrm{~mm}$ ) of a focal electrical stimulation applied on the cortical surface [4, 5, 6, 7]. For the classical referenced mode, the signal is measured between each active electrode and a distant reference electrode or an average of electrodes' signals. In this context, the DCR appears to be negative. For a differential recording mode, the signal is measured between 2 nearby active electrodes to improve spatial resolution. In this case, the DCR can either be negative or positive depending on the location of the potential towards active and reference electrodes [6]. Its amplitude generally peaks between 15 and $25 \mathrm{~ms}$ after the stimulation onset. It has been suggested that the DCR mainly depends on postsynaptic events. A weak stimulus evokes a 20 ms negative deflection called the primary negative potential (N1) that represents excitatory post-synaptic potentials of apical dendrites [5]. A stronger stimulus intensity is supposed to elicit responses in which spikes of positive polarity and brief duration (approximately $5 \mathrm{~ms}$ ) appear at the initial phase of the response, and are followed by N1. These brief positive spikes have been ascribed to serial "all or none" discharges in the soma of cortical pyramidal neurons and could be illustrative of the summation of synchronous action potentials [5]. The early positive deflection is called the P0 component.

For bipolar DES applied on the cortical surface at a given intensity, the depth of the electrical field is determined by the inter-electrode distance (IED). The longer the IED is, the deeper in the cortex the electrical field is. It has been 
demonstrated that large-diameter axons in the subcortex are the most excitable neural elements when electrical fields are generated and extend from the cortical surface to the subcortical white matter [8]. In addition, when the delivered current intensity is increased, the electrical field penetrates deeper into the cortex. It has been demonstrated experimentally and theoretically through modeling, that the generation of action potentials in an axon is obtained with less charge injections when the Pulse Width (PW) is shorter than the chronaxy [9, 10]. The relationship between the charges injected to reach the threshold and the PW is not flat, but roughly an affine function with a positive slope (see figure 7 of [2]). The more the PW decreases the more the required charges to reach the threshold decrease.

Hypothetically, reducing the PW could facilitate the generation of action potentials in pyramidal neurons of deep cortical layers that project to remote cortical areas through subcortical white matter. In the literature reporting CCEPs triggered and measured with grids of $1 \mathrm{~cm}$-spaced electrodes, PWs are inferior to those commonly used during intraoperative mapping with classical $5 \mathrm{~mm}$-spaced bipolar Ojemann's stimulation [11]. The effects of PW and interelectrodes spacing on EPs in the context of DES should be disambiguated. This work is a first attempt to clarify this issue as we manipulated these two parameters in order to determine whether the P0 increases when the inter-electrode distance is increased and the PW is decreased.

\section{METHODS}

\section{A. Patient}

This paper reports the case of a right-handed 50-years old woman who underwent a surgery for a low-grade glioma located within the left paralimbic region with frontal predominance and a beginning of contralateral extension. The present study protocol was approved by the Ethics Committee of the University Hospital Center of Montpellier (protocol: UF 965, $\mathrm{n}^{\circ}$ 2014-A00056-43). The patient gave its signed written informed consent to participate in the study.

\section{B. Electrophysiological recordings}

Electro-corticographic (ECoG) data were recorded intraoperatively after the tumor resection, under general anesthesia. Two 4-contacts strips of ECoG electrodes (2.5 $\mathrm{mm}$ exposed surface platinium contacts, $10 \mathrm{~mm}$ spaced, DIXI, France) were positioned on the surface of the brain (Fig. 1B). The first strip was placed on the superior temporal gyrus and the second on the dorsolateral prefrontal cortex (electrode numbers range from 1 to 4 and from 5 to 8 respectively, as presented in Fig. 1B). ECoG signals were recorded using differential configurations for both strips, which means that the anode and cathode were two adjacent electrodes. A cup-electrode $(A u)$ located on the left mastoid (ipsilateral to surgery) served as reference for the 4th electrode of each strip (i.e. electrodes 4 and 8, as seen in Fig. $1 \mathrm{~B}$, were recorded on common mode). Importantly, this method is not classically used but is less sensitive to noise by being independent from the positioning of a common reference electrode. ECoG signals were sampled at $10 \mathrm{kHz}$ (PowerLab, ADInstrument) and band-pass filtered from 0.5
$\mathrm{Hz}$ to $1 \mathrm{kHz}$. The front-end amplifier (g.BSamp, G.tec, Austria) was grounded to a patch-electrode located on the left acromion and signals were recorded with a gain of 1,000 to avoid saturation.

Importantly, the $50 \mathrm{~Hz}$ notch filter integrated in the amplifier was turned off for the first strip ("ECoG 1" in Fig. 1B). This was used to avoid the prominent filter impulse response on recordings, which appears as a damped sine wave and requires additional post-processing. Removing the notch filter for the first strip allowed real-time observation of the early P0 component at the expense of significant and irregular signal contamination by the $50 \mathrm{~Hz}$ electrical noise. The notch filter was kept activated for the other strip ("ECoG 2 " in Fig. 1B) allowing noise-free signal recordings and thus easier and more robust real-time observation of the late N1 component. Unused amplifier's channels were short-circuited to ground.

\section{DES and anatomical sites of stimulation}

Usual cortical and white matter mappings were completed on the awake patient. DES was delivered thanks to a bipolar probe $(0.5 \mathrm{~mm}$ diameter electrode tips, $5 \mathrm{~mm}$ apart) (Nimbus, Innopsys, France). Biphasic constant-current square wave pulse of $1 \mathrm{~ms}$ duration was applied at a fixed frequency of 60 $\mathrm{Hz}$. The current intensity was set to $2 \mathrm{~mA}$. Brain mapping is presented in Fig. 1A. Functional sites were identified during the awake phase: cortical stimulations on tag 1 induced involuntary hemifacial movements and identified the primary motor area of the face. Stimulations on tags 2 and 3 led to complete anarthria and stopped movements of the contralateral upper limb respectively, identifying the ventral premotor cortex. Stimulation on tag 4, (precentral gyrus) also generated articulatory troubles. Stimulations on tag 5 (dorsolateral prefrontal cortex) and 50 (superior temporal region) led to complete anomia. At the bottom of the cavity, the insular surface was exposed and the inferior frontooccipital fasciculus identified: stimulations on tags 47 and 48 led to reproducible semantic paraphasia.

To study more precisely the electrophysiological effects of DES, we selected 3 functional sites, further studied under general anesthesia: (1) on tag 4 (near electrode 6); (2) on tag 50 (near electrode 1); (3) on tags 47-48 (subcortically). At each stimulation site, 4 stimulation conditions were tested as presented in Tab. 1. Each one was performed twice consecutively; after the tumor resection. The frequency of 9 $\mathrm{Hz}$ was chosen because it allowed long enough time-window (111ms) to record the N1 component, without requiring overly extended stimulation time for averaging purposes.

TABLE I. DES CONDITIONS

\begin{tabular}{|l|l|l|l|l|}
\hline & Freq. & Intensity & Pulse width & \multicolumn{1}{|c|}{$\begin{array}{c}\text { Inter-Electrode } \\
\text { Distance }\end{array}$} \\
\hline Condition 1 & $60 \mathrm{~Hz}$ & $1.5 \mathrm{~mA}$ & $1.0 \mathrm{~ms}$ & $5 \mathrm{~mm}$ \\
\hline Condition 2 & $9 \mathrm{~Hz}$ & $1.5 \mathrm{~mA}$ & $1.0 \mathrm{~ms}$ & $5 \mathrm{~mm}$ \\
\hline Condition 3 & $9 \mathrm{~Hz}$ & $1.5 \mathrm{~mA}$ & $0.5 \mathrm{~ms}$ & $5 \mathrm{~mm}$ \\
\hline Condition 4 & $9 \mathrm{~Hz}$ & $1.5 \mathrm{~mA}$ & $0.5 \mathrm{~ms}$ & $10 \mathrm{~mm}$ \\
\hline
\end{tabular}




\section{Evoked potentials}

Mean EPs traces were obtained by averaging ECoG signals time-locked to the DES onset, upon a time window equals to the period of stimulation $(111 \mathrm{~ms}$ at $9 \mathrm{~Hz}$ and $16 \mathrm{~ms}$ at $60 \mathrm{~Hz}$ ). The number of stimuli available depends on the duration of the stimulation provided by the surgeon upon the tested area. In our case, stimulation durations ranged from 4 to $8 \mathrm{~s}$.

\section{RESULTS}

DCRs were recorded on multiple sites and for various stimulation conditions on both electrodes. However, only recordings made between electrodes 2 and 3 of strip 1 ("EcoG 1" in Fig. 1B) are presented as they showed systematic DCR when DES was applied on tag 50 with good signal quality throughout the experiment. The number of stimuli available ranged from 52 to 65 for $9 \mathrm{~Hz}$ DES and we systematically used the first 50 stimuli for averaging. Similarly, for the $60 \mathrm{~Hz}$ DES, the number of stimuli ranged from 254 to 298 and only the first 250 stimuli were kept. Mean DCRs were thus obtained by averaging EPs for each
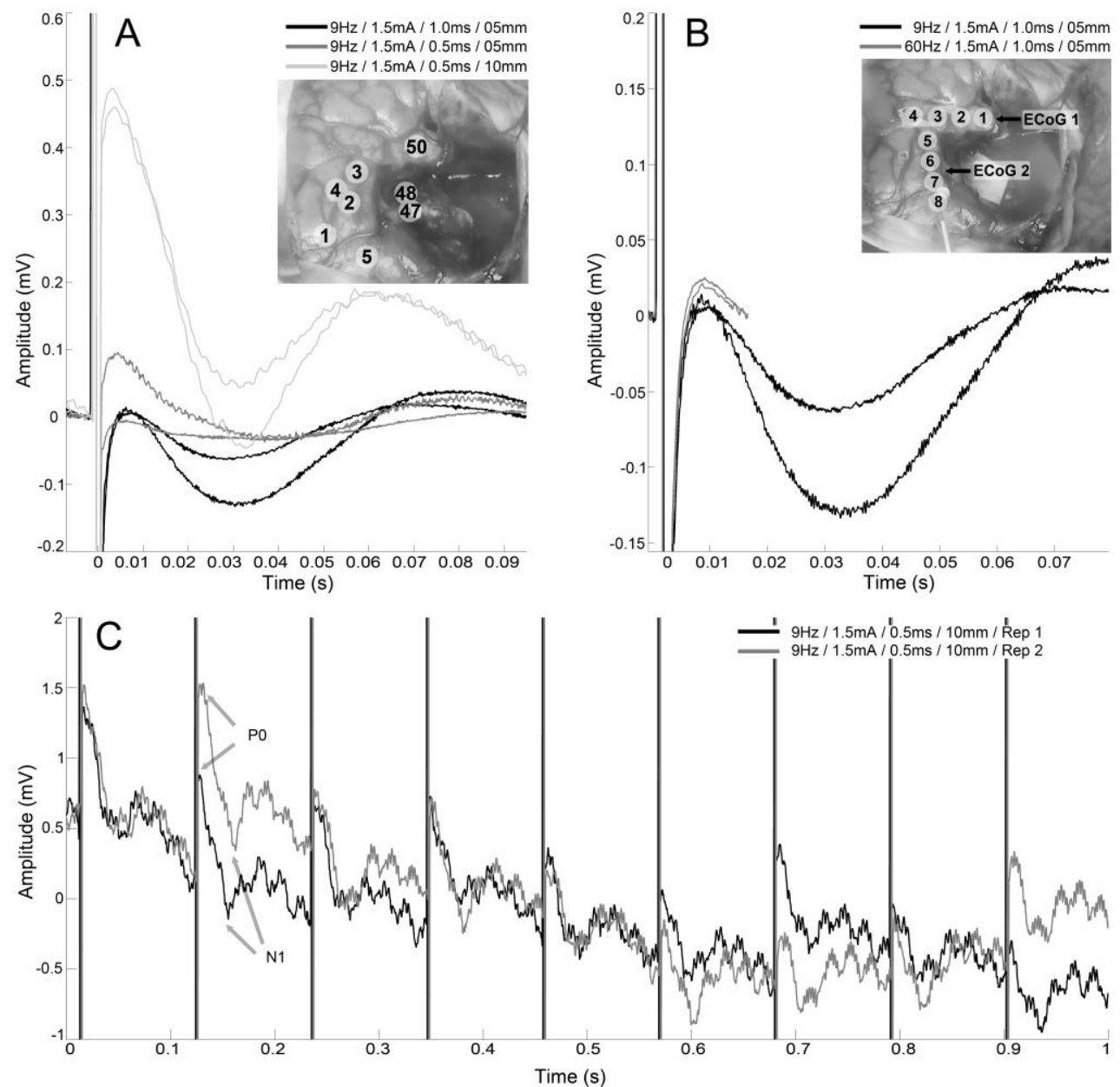

Figure 1. A: Mean DCR over 50 stimuli for the 3 conditions at $9 \mathrm{~Hz}$ (each condition was repeated twice), recorded between electrodes 2 and 3 . The average P0 latency is $3.8 \mathrm{~ms}$ and N1 latencies range from 28 to $39 \mathrm{~ms}$. DES was applied cortically on tag 50 (near the electrode 1 of the first ECoG strip). B: Mean DCR over $50(9 \mathrm{~Hz})$ and $250(60 \mathrm{~Hz})$ stimuli for the 9 and $60 \mathrm{~Hz}$ DES conditions using similar parameters $(\mathrm{I}=1.5 \mathrm{~mA}$, PW=1ms, IED=5mm, each condition was repeated twice), recorded between electrodes 2 and 3. DES was applied cortically on tag 50 (near the electrode 1 of the first ECoG strip). C: Subset of the raw ECoG signal during $9 \mathrm{~Hz}$ DES on tag 50 with parameters: $\mathrm{I}=1.5 \mathrm{~mA}, \mathrm{PW}=0.5 \mathrm{~ms}$, IED=10mm, recorded between electrodes 2 and 3 . Both repetitions are presented as "Rep 1" and "Rep 2". The magnified P0 but also the N1 components can be observed after each stimulation artifact. 


\section{DISCUSSION}

It is assumed that the DCR has different profiles which are correlated to the local cytoarchitectonic organization of the stimulated area [5]. These profiles could be used to establish, in real-time and in vivo, the electrophysiological state of the stimulated cortical column in terms of excitability [6].

Decreasing the PW and increasing the IED should activate neural elements with less charge injections and allow the current field to reach pyramidal somas and/or axons in deeper cortical layers with a higher intensity and even subcortical white matter [7]. According to this hypothesis, we varied these two parameters and were clearly able to trigger a magnified P0, suggesting an initial summation of synchronous action potentials (not a summation of excitatory postsynaptic potentials as for the N1 component). Measuring $\mathrm{P} 0$ could be of great value in order to determine whether DES induces action potentials that propagate to other remote cortical areas.

A prediction would be that CCEP should be more frequently observed when a significant P0 is observed in DCR (assuming that the two cortical sites are anatomically connected). Logically, P0 should also be visible on ACEP since in this case, subcortical axons are stimulated and thus action potentials are triggered, propagating towards the cortical surface. This is the case in [7] (see Fig. 2, note that P0 was negative because of a differential recording mode) and [12] (see Fig. 5 with different stimulation parameters) but could not be observed in [13] because of durable stimulation artifacts (see Fig. 4, similar stimulation parameters as for [7]). However, the distant summation of action potentials (P0) in ACEP and CCEP could be limited due to the divergence of projection pathways from the stimulation site. Regarding DCR, the summation could be more important due to the activation of all the fibers in the vicinity of DES.

DES can be used to probe the spatiotemporal connectivity and dynamics of short- or long- range networks when combined with electrophysiological recordings. This approach has been used for pre-surgical planning of drugresistant epileptic patients, by using ECoG and a grid implanted at the surface of the grey matter [11]. However, the effects of DES at higher frequencies remain unclear. It has been demonstrated that $20 \mathrm{~Hz}$ DES induces slow negativities in separated DCRs, which fuse to produce a progressive negative shift in the baseline of the ECoG signal. At the same time as this shift develops, the amplitude of sequential DCRs decreases [14]. It was proposed that certain neural elements could not recover fast enough from DES and saturate because of the summation of DCRs. Hence, higher stimulation frequencies might induce local and progressive hyperpolarisation in the vicinity of the stimulated area, which may perturb its global functioning [7]. This might be an explanation for the use of higher frequencies (i.e. $50-60 \mathrm{~Hz}$ ) during cortical mapping as the stimulation period (i.e. 16 to $20 \mathrm{~ms}$ ) is close to the DCR duration (about 15 to $20 \mathrm{~ms}$ ). Similarly, we noticed a slight decrease in the amplitude of the P0 component over time during $9 \mathrm{~Hz}$ DES. However, physiological reasons remain unclear although membrane saturation could also be at the origin of weakened summation of action potentials.

\section{CONCLUSION}

P0 component, may be indicative of the spread of action potentials onto white matter tracts towards remote cortical areas. From a methodological perspective, P0 is of particular interest because it contrasts with N1 whose latency does not concord with the stimulation period when using usual cortical mapping frequencies. However, we did not observe the P0 component for $60 \mathrm{~Hz}$ DES condition, suggesting that there is no spread of electrophysiological activity in this particular case. Further manipulations are required in order to clarify whether and how classical uses of $60 \mathrm{~Hz}$ DES induce effects in remote cortical areas and decipher the electrophysiological implications of the clinical use of DES [7].

\section{REFERENCES}

[1] H. Duffau, "Lessons from brain mapping in surgery for low-grade glioma: insights into associations between tumour and brain plasticity", Lancet Neurol., vol. 4, no. 8, pp. 476-486, Aug. 2005.

[2] M. Vincent, O. Rossel, M. Hayashibe, G. Herbet, H. Duffau, D. Guiraud, F. Bonnetblanc, "The difference between electrical microstimulation and direct electrical stimulation - towards new opportunities for innovative functional brain mapping?", Rev. Neurosci., vol. 27, no. 3, pp. 231-258, Apr. 2016.

[3] M. Vincent, O. Rossel, B. Poulin-Charronnat, G. Herbet, M. Hayashibe, H. Duffau, D. Guiraud and F. Bonnetblanc, "Case report: Remote neuromodulation with direct electrical stimulation of the brain, as evidenced by intra-operative EEG recordings during wide-awake neurosurgery", Clin. Neurophysiol., vol. 127, no. 2, pp. 1752-1754, Feb. 2016.

[4] E.D. Adrian, "The spread of activity in cerebral cortex", J. Physiol., vol. 88, no. 2, pp. 127-161, Nov. 1936.

[5] S. Goldring, G.W. Harding, E.M. Gregorie, "Distinctive electrophysiological characteristics of functionally discrete brain areas: a tenable approach to functional localization", J. Neurosurg., vol. 80, no. 4, pp. 701-709, Apr. 1994.

[6] M. Vincent, O. Rossel, H. Duffau, F. Bonnetblanc, D. Guiraud, "A measure of cortico-cortical potentials evoked by $10 \mathrm{~Hz}$ direct electrical stimulation of the brain and by means of a differential recording mode of electrocorticographic signals", Conf. Proc. IEEE Eng. Med. Biol. Soc., pp. 4543-4546, Aug. 2016.

[7] M. Vincent, D. Guiraud, H. Duffau, E. Mandonnet, F. Bonnetblanc, "Electrophysiological brain mapping: Basics of recording evoked potentials induced by electrical stimulation and its physiological spreading in the human brain", Clin. Neurophysiol., vol. 128, no. 10, pp. 1886-1890, Oct. 2017.

[8] W.M. Landau, G.H. Bishop, M.H. Clare, "Site of excitation in stimulation of the motor cortex", J. Neurophysiol., vol. 28, no. 6, pp. 1206-1222, Nov. 1965.

[9] D.B. McCreery, W.F. Agnew, T.G.H. Yuen, L.A. Bullara, "Charge density and charge per phase as cofactors in neural injury induced by electrical stimulation", IEEE Trans. Biomed. Eng., vol. 37, no. 10, pp. 996-1001, Oct. 1990.

[10] D.R. Merrill, M. Bikson, J.G.R. Jefferys, "Electrical stimulation of excitable tissue: design of efficacious and safe protocols", J. Neurosci. Methods, vol. 141, no. 2, pp. 171-198, Feb. 2005.

[11] R. Matsumoto, D.R. Nair, E. LaPresto, I. Najm, W. Bingaman, H Shibasaki, H.O. Lüders, "Functional connectivity in the human language system: a corticocortical evoked potential study", Brain, vol. 127, no. 10, pp. 2316-2330, Oct. 2004.

[12] Y. Yamao, R. Matsumoto, T. Kunieda, Y. Arakawa, K. Kobayashi, K Usami, S. Shibata, T. Kikuchi, N. Sawamoto, N. Mikuni, A. Ikeda, H. Fukuyama and S. Miyamoto, "Intraoperative dorsal language network mapping by using single-pulse electrical stimulation", Human Brain Mapp., vol. 35, no. 9, pp. 4345-4361, Sep. 2014.

[13] E. Mandonnet, Y. Dadoun, I. Poisson, C. Madadaki, S. Froelich and P. Lozeron, "Axono-cortical evoked potentials: A proof-of-concept study", Neurochirurgie, vol. 62, no. 2, pp. 67-71, Apr. 2016.

[14] S. Goldring, M.J. Jerva, T.G. Holmes, J.L. O’Leary, J.R. Shields, "Direct response of human cerebral cortex", Arch. Neurol., vol. 4, pp. 590-598, Jun. 1961. 
